\title{
Left Atrial Thrombus Causing Pulmonary Embolism by Passing Through an Atrial Septal Defect
}

\author{
Yukako Ishihara, MD; Hidehiko Hara, MD; Tomokatsu Saijo, MD; Atsushi Namiki, MD; \\ Makoto Suzuki, MD; Hironori Hirai, MD; Tetsu Yamaguchi, MD
}

\begin{abstract}
A 66-year-old woman admitted with dyspnea on exertion had atrial fibrillation and left ventricular dysfunction. Echocardiography revealed an atrial septal defect (ASD) and a soft, easily deformable thrombus in the dilated left atrium. The atrial mass suddenly disappeared on the 10th day after admission, and contrast-enhanced chest computed tomography and pulmonary blood flow scintigraphy showed that the thrombus had detached from the left atrium, floated into the right atrium through the ASD and caused pulmonary embolism. This is the first documented case of a left atrial thrombus causing pulmonary embolism by passing through an ASD. When an ASD is present, it is important to consider not only paradoxical thromboembolism (from the right to the left atrium), but also pulmonary embolism caused by thromboembolism from the left to the right atrium. (Circ J 2002; 66: 109-110)
\end{abstract}

Key Words: Atrial fibrillation; Atrial septal defect; Left atrial thrombus; Pulmonary embolism

$\mathbf{L}$ eft atrial thrombus sometimes accompanies atrial fibrillation (AF) and in the absence of rheumatic valvular disease, is associated with a 5- to 7 -fold increased risk of ischemic stroke caused by the thrombus; ${ }^{1}$ the rate has been reported to be $6.3 \%$ per year! Furthermore, left atrial thrombi can also embolize to abdominal or pelvic organs and in Japan, it has been reported that the annual incidence of thromboembolic complications is $2.1 \%$ in non-valvular heart disease? However, there are no reports in the literature of left atrial thrombus causing pulmonary embolism by passing through an atrial septal defect (ASD) and we present the first case in which a left atrial thrombus caused by AF embolized to the pulmonary artery via an ASD, causing pulmonary embolism.

\section{Case Report}

A 66-year-old Japanese woman was admitted with dyspnea on exertion. She had a history of a hepatic disorder at 62 years of age. Her symptoms had been getting worse for more than 1 year, and the degree of dyspnea on admission corresponded to New York Heart Association grade III. Her blood pressure was $142 / 90 \mathrm{mmHg}$ and her heart rate was irregular and about 150 beats/min at the time of admission. Physical examination did not detect jugular venous distention, hepatomegaly or splenomegaly, but there was pretibial edema. Auscultation of the chest revealed slight bilateral inspiratory crackles at the lung bases and a grade III/VI systolic murmur along the left sternal border of the second intercostal space. Blood testing showed no specific abnormalities, but arterial blood gas analysis while the

(Received January 9, 2001; revised manuscript received April 26, 2001; accepted May 18, 2001)

Third Department of Internal Medicine, Toho University School of Medicine, Ohashi Hospital, Tokyo, Japan

Mailing address: Atsushi Namiki, MD, Third Department of Internal Medicine, Toho University School of Medicine, Ohashi Hospital, 217-6 Ohashi, Meguro-ku, Tokyo 153-8515, Japan. E-mail: namiki@ oha.toho-u.ac.jp patient was on nasal oxygen $(2 \mathrm{~L} / \mathrm{min})$ revealed a $\mathrm{PaO}_{2}$ of $66.0 \mathrm{mmHg}$ and $\mathrm{PaCO}_{2}$ of $33.0 \mathrm{mmHg}$. Her electrocardiogram showed $\mathrm{AF}$ and poor $\mathrm{R}$ wave progression in $\mathrm{V}_{1-3}$. The chest radiograph showed pulmonary congestion and bilateral pleural effusions.

Transthoracic echocardiography revealed a highly echogenic mobile mass $(24 \times 17 \mathrm{~mm})$ in the dilated left atrium (left atrial diameter: $57 \mathrm{~mm}$; Fig 1), and diffuse left ventricular hypokinesis (ejection fraction: 47\%; fractional shortening: $21 \%$ ) without left ventricular dilation (left ventricular end-diastolic diameter: $49 \mathrm{~mm}$; left ventricular end-systolic diameter: $38 \mathrm{~mm}$ ). The right atrium and right ventricle were mildly dilated, and shunt flow from the left atrium to the right atrium was clearly detected by color Doppler echocardiography, suggesting the existence of an ASD. Mild mitral and tricuspid regurgitation were diagnosed, but we did not find aortic or pulmonary regurgitation. Transesophageal echocardiography clearly showed an ASD $(8.2 \mathrm{~mm}$ in maximum diameter; Fig 2). The mass appeared to adhere to the

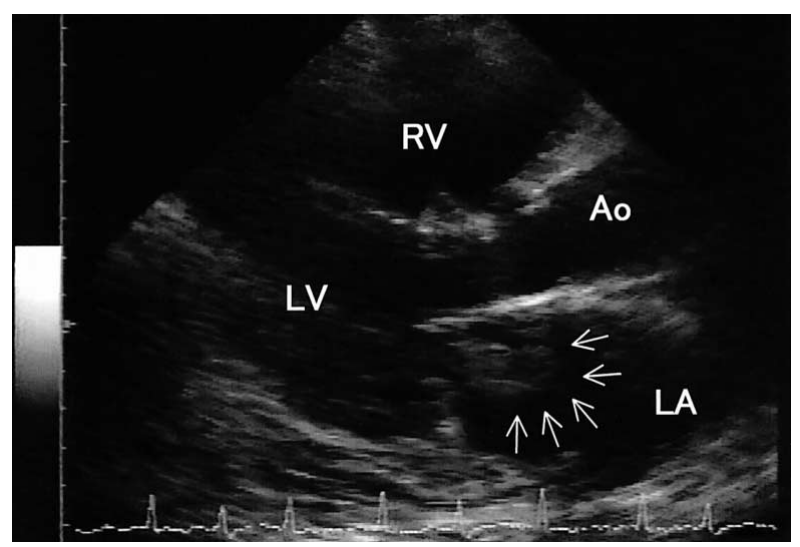

Fig 1. Transthoracic echocardiogram (long-axis view) showing a mobile echogenic mass $(24 \times 17 \mathrm{~mm})$ (arrows). LA, left atrium; LV, left ventricle; Ao, aorta; RV, right ventricle. 


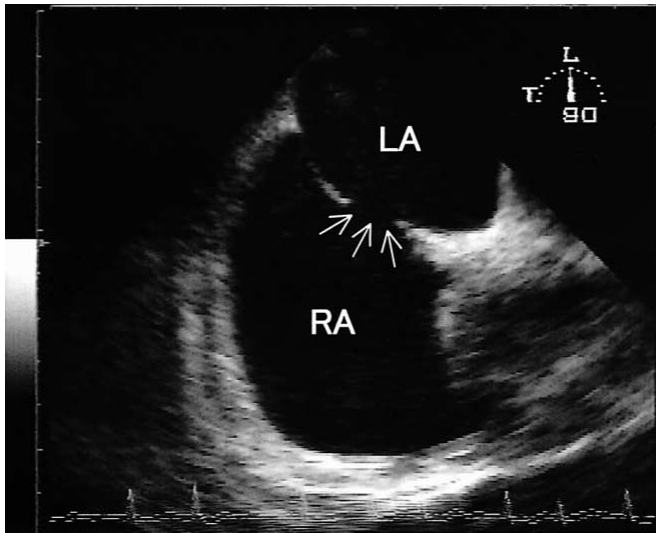

Fig 2. Transesophageal echocardiogram (longitudinal view) showing the atrial septal defect $(8.2 \mathrm{~mm}$ in diameter) (arrows). LA, left atrium; $\mathrm{RA}$, right atrium.

left atrial appendage, but was mobile in the left atrium. It sometimes moved into the left ventricle through the mitral valve. Spontaneous echo contrast did not occur in the left atrium on transthoracic echocardiography or on transesophageal echocardiography, but we considered that the mass was a thrombus formed by AF and began warfarin therapy to maintain an INR between 2.5 and 3.0.

The size and form of the atrial mass did not change on serial echocardiography until the 10th day after admission when it suddenly disappeared, and it was thought that the thrombus had detached and embolized to another organ. Computed tomography (CT) did not detect any infarction in the brain or abdominal or pelvic organs. Contrast-enhanced chest CT showed an embolus in the left pulmonary artery (A9) and pulmonary blood flow scintigraphy also revealed a defect in the left $\mathrm{S} 9$ area. We hypothesized that the thrombus had detached from the left atrium, floated into the right atrium through the ASD, and then caused pulmonary embolism. We continued the anticoagulation therapy, and follow-up contrast-enhanced chest CT and pulmonary blood flow scintigraphy showed that the thrombus in the left A9 pulmonary artery had disappeared and that the defect in the left segment 9 area had resolved. We performed cardiac catheterization before discharge and found that pressure of both the right and left atria was not elevated (pulmonary capillary wedge pressure: $8 \mathrm{mmHg}$; pulmonary arterial pressure: $29 / 16 \mathrm{mmHg}$; right atrial pressure: $7 \mathrm{mmHg}$ ). Pulmonary blood flow/systemic blood flow (Qp/Qs) was 1.88 , and the shunt ratio from left to right was $47 \%$.

\section{Discussion}

Many reports have described thromboemboli passing through an ASD. In many cases, the thrombus formed in the deep veins of the lower extremities, floated into the right atrium and went into the left atrium via the ASD, causing paradoxical thromboembolism in the brain or abdominal organs. Although fewer than 40 cases have been reported, the incidence is probably much higher? Among patients with stroke, the prevalence of an ASD (eg, patent foramen ovale) was 54\% in patients with no identifiable cause for the stroke? In the present patient, it is likely that thrombus formed in the left atrium because of AF, passed to the right atrium via the ASD and then caused the pulmonary embolism. Fortunately, the obstruction to the pulmonary arteries was not very large and caused limited signs and symptoms.

To the best of our knowledge, this is the first report of pulmonary embolism caused by a thrombus in the left atrium that passed through an ASD into the right atrium. The maximum diameter of the ASD was $8.2 \mathrm{~mm}$ on echocardiography and the shunt ratio from left to right was $47 \%$. The size of the thrombus in the left atrium was about $24 \times 17 \mathrm{~mm}$, greater than the maximum diameter of the ASD, but it appeared to be soft and its shape changed while moving to and fro in the left atrium, which may be the reason why it was able to pass into the right atrium via the ASD.

Recurrent pulmonary emboli caused by left atrial myxoma embolization via an ASD has been reported5 It has also been reported that left atrial myxoma can cause pulmonary infarction by occluding the pulmonary veins. In the present patient, there was no possibility that the mass was myxoma because the embolus disappeared with warfarin therapy. Complications of AF and left atrial dilation and the existence of a stem connected to the left atrial appendage also suggest that the mass was a thrombus, not myxoma. An important question is why there have not been other reports of left atrial thrombus causing pulmonary embolism via an ASD. We hypothesize that a thrombus rarely forms in the left atrium when an ASD is present because the left to right blood flow through the defect is high. The left atrium often is small in patients with ASD. In the present patient, concomitant AF and left ventricular dysfunction might have caused left atrial dilation, which then permitted the formation of a thrombus that dislodged and floated into the pulmonary artery after passing through the ASD. Information relating to hereditary or acquired hypercoagulation diathesis would have been interesting, but was not investigated sufficiently before starting warfarin therapy. However, because this patient was 66 years old and had not suffered from any other thromboembolic disease, there is little likelihood of hereditary hypercoagulation diathesis. We did test for lupus anticoagulant and antinuclear antibody for acquired hypercoagulation diathesis, but those results were negative.

\section{References}

1. Stroke Prevention in Atrial Fibrillation Investigators. Stroke prevention in atrial fibrillation study: Final results. Circulation 1991; 84: 527-539.

2. Toda G, Akiyama K, Sakuragawa K, Iliev II, Hayano M, Yano K. Thromboembolic complication in atrial fibrillation in a long-term follow-up: The relationship with underlying disease, type of atrial fibrillation, and antithrombotic therapy. Jpn Circ J 1998; 62: $255-$ 260.

3. Aburahma AF. Work-up and management of patients with paradoxical emboli. WV Med J 1996; 92: 260-264.

4. Lechat PH, Mas JL, Lascault G, Loron PH, Theard M, Klimczac M, et al. Prevalence of patent foramen ovale in patients with stroke. $N$ Engl J Med 1988; 318: 1148-1152.

5. Seagle RL, Nomeir A-M, Watts LE, Mills SA, Means WE. Left atrial myxoma and atrial septal defect with recurrent pulmonary emboli. South Med J 1985; 78: 992-994.

6. Stevens LH, Hormuth DA, Schmidt PE, Atkins S, Fehrenbacher JW. Left atrial myxoma: Pulmonary infarction caused by pulmonary venous occlusion. Ann Thorac Surg 1987; 43: 215-217. 